\title{
Development of Construction Performance Monitoring Methodology using the Bayesian Probabilistic Approach
}

\author{
Yongho $\mathrm{Ko}^{1}$ and Seungwoo Han ${ }^{2}$
}

\author{
${ }^{1}$ Master Candidate, Department of Architectural Engineering, Inha University, Korea \\ ${ }^{2}$ Professor, Department of Architectural Engineering, Inha University, Korea
}

\begin{abstract}
Process planning is based on engineers' experience. This often results in cost overruns and delays. Precise planning based on reliable data is required to avoid these issues. Accurate productivity prediction is one of the most important issues in process planning. However, it has been shown that the process of collecting enough data has severe limitations in terms of time and human resources. Numerous studies have attempted to overcome the difficulties of accurate productivity prediction. This paper proposes a methodology for monitoring construction projects by implementing a database that is developed using the Bayesian posterior -probabilistic method. This method is considered to be appropriate in civil engineering because it provides reliable probabilities by presenting updated results based on continuously collected data rather than focusing on a one-time observation and associated analysis. This paper focuses on high-rise, curtain wall operations as an example for database implementation and Bayesian application. The results obtained using the Bayesian method illustrate appropriate convergence to specific mean values with small variances of standard deviations. This result shows that a Bayesian probabilistic approach can be utilized in civil engineering, to provide more accurate prediction results based on updated information.
\end{abstract}

Keywords: Bayesian; performance monitoring system; construction data; database; information technology

\section{Introduction \\ 1.1 Aim and Objectives}

In recent times, construction projects have become larger, and the construction process has become more complicated (Kim and Kim 2010). According to a statistical report from the Korean Ministry of Land, Transport and Maritime Affairs, 144 high-rise construction projects were completed in 2009, and 243 in 2010 (The Korean Ministry of Land 2011). In order to successfully complete a high-rise construction project, precise and effective process management is needed. However, studies show that performance analysis and process management in the construction industry are not as effective as in the manufacturing industry (Navon 2005). This occurs because the decision-making process at construction sites is based on the experience of the site engineers. Such decision making is not objective, and sometimes leads to cost overruns and delays (Lim 2009). Therefore, there is a need to analyze actual data before any decisions are made. However, it is difficult to analyze data

*Contact Author: Seungwoo Han, Professor,

Inha University, 100 Inha-ro, Nam-gu,

Incheon 402-751, Korea

Tel/Fax: +82-32-860-7595

E-mail: shan@inha.ac.kr

(Received April 6, 2014 ; accepted October 15, 2014 ) consistently in the construction site environment, because of frequently changing conditions.

A paper by Han et al. (2014) presented database structures as a solution to overcome these limitations. The database framework suggested in the paper (Han et al. 2014) provides the fundamental design and basic structures to assist site personnel in making reasonable decisions. To develop this database and expand its applications to include more practical operations, a new methodology to update the collected data is required. The available information may need to be updated in real time or at a specific time interval. Thus, it is required that results in a database effectively reflect the updates made, in order to provide users with more reliable and realistic information. The Bayesian probabilistic approach has been generally used for estimating parameters on distributions changed by new information in science and engineering. (Ang and Tang 2007; Huang and McBean 2008). Accordingly, this paper suggests a methodology to compare the planned and actual performances, by means of a database technique and the Bayesian posterior probability approach.

\subsection{Methodology and Scope}

This paper focuses on the unit curtain wall system as a sample process considered as one of the most critical construction operations that have increased in high rise building construction projects recently. 
A flowchart illustrating the overall research process in this study is shown in Fig.1. As illustrated in Fig.1., the overall process of this research is composed of six steps; literature reviews, data collection, feasibility and application of the Bayesian posterior probabilistic approach, database development, and conclusions and discussions. The Bayesian posterior probabilistic approach is applied with actual datasets after checking the reliability of the method through a pre-test with randomly generated datasets. The feasibility and reliability on the database established based on the Bayesian method would be continuously checked with new collected datasets.

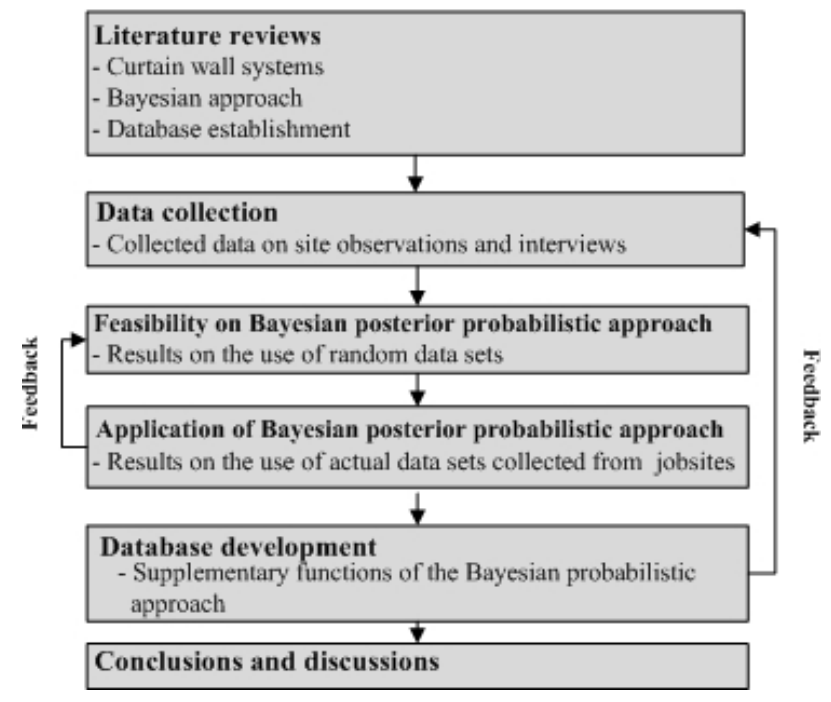

Fig.1. Flowchart of Research Process

Detailed descriptions of each step are described as follows:

1) The paper presented the fundamental descriptions of curtain wall operations, and introduced the operating procedures of the unit curtain wall system.

2) The literature related to the Bayesian probabilistic approach and databases for performance monitoring has been studied.

3) Actual datasets from the targeted jobsites have been collected and interviews with site personnel have been conducted to supplement information.

4) The feasibility of Bayesian probabilistic approaches using random data generated by Microsoft Excel 2010 has been tested. This verification became a pre-investigation for the actual testing and a pre-test for wider applications.

5) The Bayesian posterior probabilistic approach has been applied to the actual dataset, for database development.

6) It is recommended that the database is implemented following the application of the Bayesian posterior probabilistic approach.

\section{Literature Review}

\subsection{High-Rise Curtain-Wall Operation}

According to the American Society of Civil Engineers (ASCE), a high-rise building is defined as a building whose height is greater than the buildings in the area, wherein uncommon techniques, and methods of construction have been applied (Choi 2006). Curtain wall operations are widely applied in such high-rise buildings, especially in urban areas. Curtain wall operations comprise $10 \%$ to $15 \%$ of the total cost of a construction project, and are always part of the critical path (Lee et al. 2011). The curtain wall operation is composed of two main systems - a unit system and a stick system. The basic difference of these two systems is derived from the place for assembling. A unit system already assembled in a factory is delivered and installed directly, whereas, a stick system is delivered and mostly assembled at jobsites. Accordingly, the unit system has been used frequently in high-rise building construction projects, which is the reason why good quality and high productivity are secured in spite of the material costs being more expensive than the stick system (Kim 2012; Son 2002). A construction site where a curtain wall unit system was applied has been visited. The units were delivered to the construction site by truck and transported by a forklift to the lifting zone. Generally, units are lifted by either a crane or a hoist. At this construction site, the units were lifted by a hoist to the installation zone and were installed by the installing crew. Table 1 . shows the operating procedure of the curtain wall unit system, which is divided into three main processes: unloading, lifting and installing (Han et al. 2014).

\subsection{Bayesian Posterior Probability}

A Bayesian statistical approach has been used as a powerful tool in many areas of science, including information science and engineering. It has become one of the best methods in civil engineering, where reliable prediction results are required (Yuen 2010).

The Bayesian posterior probabilistic method has been applied for delivering significant prediction results with updated information in various areas (Johnson and Wichern 2007). The textbook by Ang and Tang introduced the basic concept of this method with appropriate examples in civil engineering. The main advantage of the practical application of the Bayesian method is that it will function as one of the powerful prediction tools in situations where data are continuously collected and have significant influence in predictions such as construction and civil engineering (Ang and Tang 2007).

In this approach, one event is defined as a prior event and another as a posterior event. By Bayes' rule, the posterior probabilities are defined as follows:

$$
P\left(\pi_{1} \mid x_{0}\right)=\frac{P\left(\pi_{1} \text { occurs and observed } x_{0}\right)}{P\left(\text { observed } x_{0}\right)}
$$

where,

$\mathrm{x}_{0}$ is the new observation

$\pi_{1}$ is the population 
Table 1. Curtain Wall Operation Procedure (Han et al. 2014)

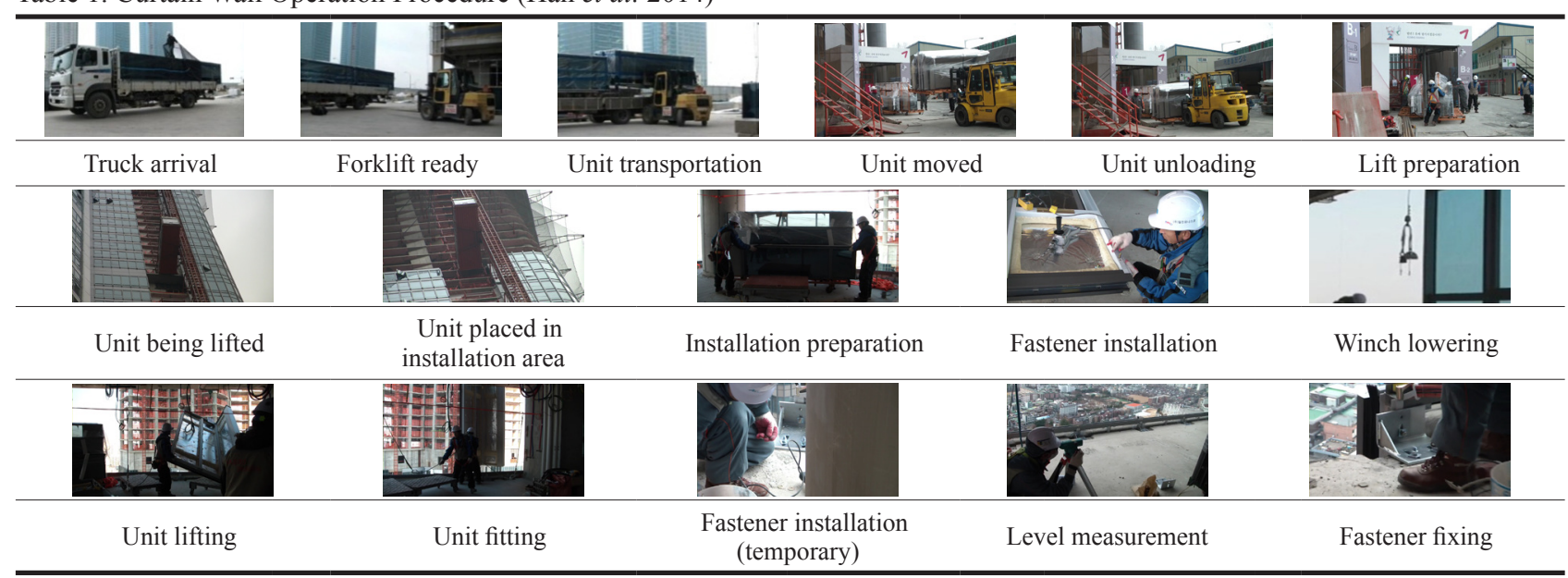

Equation (1) can be redefined as follows.

$\mathrm{P}\left(\right.$ observed $\left.\mathrm{x}_{0} \mid \pi_{1}\right) \mathrm{P}\left(\pi_{1}\right)$

$\overline{\mathrm{P}\left(\text { observed } \mathrm{x}_{0} \mid \pi_{1}\right) \mathrm{P}\left(\pi_{1}\right)+\mathrm{P}\left(\text { observed } \mathrm{x}_{0} \mid \pi_{2}\right) \mathrm{P}\left(\pi_{2}\right)}$

$$
=\frac{p_{1} f_{1}\left(x_{0}\right)}{p_{1} f_{1}\left(x_{0}\right)+p_{2} f_{2}\left(x_{0}\right)}
$$

The definition of the posterior probability is as follows:

$$
P\left(\pi_{2} \mid x_{0}\right)=\frac{p_{2} f_{2}\left(x_{0}\right)}{p_{1} f_{1}\left(x_{0}\right)+p_{2} f_{2}\left(x_{0}\right)}
$$

Research by Soibelman and Kim (2002) suggests the Bayesian approach to be used in the identification of data features in the establishment of knowledge discovery in databases. (Soibelman and Kim 2002).

Studies in which the Bayesian probabilistic approach has been applied in construction and civil engineering indicates that this method has been adopted in two categories: 1) risk assessment and management, and 2) status assessment of structures and materials. Referring to risk assessment and management, the Bayesian approach was mostly used for analysis and prediction based on survey results (Kim and Reinschmidt 2009; Lee et al. 2009; Leu and Chang 2013; Truong 2009).

Lee suggested a methodology of risk management using a Bayesian belief network based on the survey, and applied it to the Korean shipbuilding industry (Lee et al. 2009). Kim and Reinschmidt suggested a forecasting methodology using the Bayesian beta S-curse method (BBM), which is more accurate than the earned value method (Kim and Reinschmidt 2009). The study by Troung suggested a methodology for predicting the likelihood of time overrun that could play a key role in project success using Bayesian belief networks (Truong 2009). Leu and Chang analyzed the primary types of accidents in steel building construction using Bayesian networks. The suggested model provides accurate site safety management capabilities by calculating the probabilities of safety risks, and further analyzing the causes of accidents based on their relationships (Leu and Chang 2013).
Regarding Bayesian approaches for studies on the status assessment of structures and materials, the methods were mostly used for empirical studies based on the collected or measured data (Gardoni et al. 2007; Huang and McBean 2008; Labuz et al. 2010).

Gardoni et al. suggested probabilistic models for computing the elastic modulus of concrete using the Bayesian approach which was used to assess the unknown model parameters based on experimental data. (Gardoni et al. 2007). Huang and McBean presented an assessment model for chlorine wall decay effects using a combination of the Bayesian approach and Monte Carlo Markov chain (Huang and McBean 2008). Labuz et al. studied the use of the Bayesian approach for determining the influence coefficients of local damage detection in beam-column connections (Labuz et al. 2010).

The Bayesian approach applied in this study uses a simple formula to further analyze a project's overall productivity, and provide the basis for the proposed project performance monitoring methodology. The Bayesian approach indicates that the distribution of current data can be modified according to data collected in the past. A parameter is a value that characterizes the properties of a group. The act of estimating a parameter is called statistical inference. Generally, statistical inference is performed according to data collected from experiments. The Bayesian statistical approach is applied not only to properties of the current data but also to past and updated data, which better explain the parameter (Kang 2011). The Bayesian approach uses a total probability theorem to estimate the parameter and can thus overcome the limitations of decision-making based on either experience, or the use of indirect information that sometimes leads to incorrect results. The Bayesian approach combines such information and yields an optimized parameter value. The equations applied in this paper are as follows (Ang and Tang 2007):

$$
\mu^{\prime \prime}=\frac{\overline{\mathrm{x}}\left(\sigma^{\prime}\right)^{2}+\mu^{\prime}\left(\frac{\sigma^{2}}{\mathrm{n}}\right)}{\left(\sigma^{\prime}\right)^{2}+\left(\frac{\sigma^{2}}{\mathrm{n}}\right)}
$$




$$
\sigma^{\prime \prime}=\sqrt{\frac{\left(\sigma^{\prime}\right)^{2}\left(\frac{\sigma^{2}}{n}\right)}{\left(\sigma^{\prime}\right)^{2}+\left(\frac{\sigma^{2}}{n}\right)}}
$$

where,

$$
\begin{aligned}
& \mu^{\prime \prime}=\text { mean of cumulative dataset. } \\
& \bar{x}=\text { mean of current dataset } \\
& \mu^{\prime}=\text { bayesian mean of previous dataset } \\
& \sigma=\text { standard deviation of current dataset } \\
& \sigma^{\prime}=\text { bayesian standard deviation of previous dataset } \\
& \sigma^{\prime \prime}=\text { standard deviation of cumulative dataset } \\
& n=\text { number of data in the current dataset }
\end{aligned}
$$

\subsection{Database Implementation in Construction}

Database methodologies are widely applied in construction sites. However, numerous studies related to database implementations for construction sites reveal limitations in obtaining reliable results and data collection (Forcada et al. 2007; Soibelman and Kim 2002; Tah et al. 1999). Forcada developed a web-based database to improve internal and external document management for small and medium-sized enterprises. The requirements for the database, such as rules on how to organize all the information related to construction projects, were deduced by conducting a survey in Spain. (Forcada et al. 2007). Tah developed a database management system to facilitate ongoing project information collation, organization, and management, to reflect the changing conditions in the construction environment (Tah et al. 1999). The database implemented in this study, however, does not consider construction data, as was done in the aforementioned studies. Instead, the proposed database deals with operation data that can be converted to productivity and unit cost data. This study examines the analysis of productivity by applying the Bayesian posterior probability to produce reliable productivity management results. The Bayesian methodology has also been implemented with random numbers, as well as actual data collected from an ongoing construction project in South Korea.

\section{Data Collection}

The data collected from construction sites have been analyzed where a curtain wall unit operation was being applied (Han et al. 2014). A stopwatch and video cameras were used to collect duration. The entire operation was observed using a video camera. The duration of each process was measured. The analysis focused on the installing process, where the duration has the highest standard deviation among three main processes as described in Chapter 2. The collected data were recalculated in terms of productivity, as shown in Table 4. The mean and standard deviation of the data are also shown in Table 4. This paper analyzes the data using the Bayesian posterior probability in order to suggest a more optimized value of productivity than the mean value.

\section{Application of Bayesian Posterior Probability}

The methodology was investigated using Microsoft Excel 2010 by applying equations (4) and (5). A normal distribution of the data was drawn to visually demonstrate the change. Four datasets of random variables were generated. The mean of each dataset is $3.55,4.63,4.20$, and 5.32, and the standard deviation of each dataset is $4.28,3.51,3.54$, and 3.65 , respectively as shown in Table 2.

Table 2. Productivity Data Using Random Data (units/h)

\begin{tabular}{c|c|c|c|c}
\hline Data & Set 1 & Set 2 & Set 3 & Set 4 \\
\hline 1 & 7.77 & 8.38 & 1.04 & 2.74 \\
\hline 2 & 0.58 & 3.61 & 4.45 & 10.65 \\
\hline 3 & 0.50 & 0.59 & 4.14 & 5.24 \\
\hline 4 & 4.29 & 1.01 & 9.34 & 3.67 \\
\hline 5 & 2.10 & 2.66 & 1.41 & 3.79 \\
\hline 6 & 4.39 & 2.00 & 5.58 & 3.55 \\
\hline 7 & 2.79 & 2.81 & 3.95 & 1.26 \\
\hline 8 & 0.74 & 3.88 & 6.32 & 11.23 \\
\hline 9 & 0.24 & 8.67 & 1.59 & 2.04 \\
\hline 10 & 14.47 & 11.40 & 0.57 & 3.99 \\
\hline 11 & 1.24 & 5.94 & 11.66 & 10.39 \\
\hline Mean & 3.55 & 4.63 & 4.20 & 5.32 \\
\hline S. D. & 4.28 & 3.51 & 3.54 & 3.65 \\
\hline
\end{tabular}

Fig.2. shows the normal distribution based on the generated productivity data. As shown in Fig.2., the application of each new dataset, converges the mean to a more optimized and reliable value. The standard deviation is large in the first dataset. Fig.2. also shows that the distributions change as additional datasets are introduced.

Table 3. shows the $\mu$ " and $\sigma$ " for each step, and compares these values with the mean and standard deviation of the data shown in Table 2. Note that the value of $\sigma$ " decreases as more data are added. The most important result is that $\mu^{\prime \prime}$ converges to an optimal value, and $\sigma^{\prime \prime}$ is expected to decrease further with the addition of data. This analysis shows that the use of a mean value in productivity analysis can produce incorrect results.

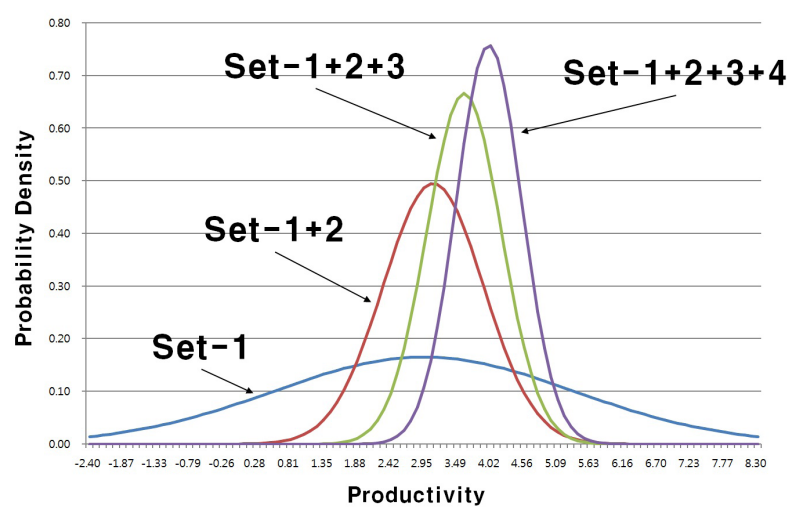

Fig.2. Normal Distribution of Datasets

After checking the fundamental feasibility of the methodology application using random datasets, the 
Table 3. $\mu$ " and $\sigma$ "Values and Comparison Using Random Data (units/h)

\begin{tabular}{c|c|c|c|c}
\hline Description & Set 1 & Set 2 & Set 3 & Set 4 \\
\hline$\mu^{\prime \prime}$ & 3.55 & 4.57 & 4.39 & 4.68 \\
\hline Mean & 3.55 & 4.63 & 4.20 & 5.32 \\
\hline$\sigma^{\prime \prime}$ & 4.28 & 1.03 & 0.74 & 0.61 \\
\hline S. D. & 4.28 & 3.51 & 3.54 & 3.65 \\
\hline
\end{tabular}

methodology has been applied to data collected from an actual construction site, focusing on the curtain wall unit system installation process. Actual datasets indicated as sets 1 to 4 were collected from four different construction sites where basic construction processes and resource information were very similar. The data collected in three months from August to October 2013, were gathered from the sites for highrise building constructions located in the Songdo district in Incheon, South Korea. Table 4. and Fig.3. show the actual productivity data from the 4 jobsites and the distributions of the collected data. For instance, set 4 in Table 4 . shows that productivity focusing on curtain wall installation was actually measured over a total of 11 days at the fourth construction site.

Table 4. Productivity Data Using Actual Data (units/h)

\begin{tabular}{c|c|c|c|c}
\hline Data & Set 1 & Set 2 & Set 3 & Set 4 \\
\hline 1 & 4.52 & 2.36 & 7.84 & 4.74 \\
\hline 2 & 4.88 & 5.50 & 8.91 & 3.59 \\
\hline 3 & 2.89 & 4.32 & 8.35 & 4.83 \\
\hline 4 & - & 3.81 & 3.41 & 3.69 \\
\hline 5 & - & 4.17 & 3.65 & 2.48 \\
\hline 6 & - & 5.46 & 9.57 & 4.00 \\
\hline 7 & - & 4.62 & 2.93 & 4.30 \\
\hline 8 & - & 4.86 & 2.77 & 4.83 \\
\hline 9 & - & - & 9.76 & 5.21 \\
\hline 10 & - & - & - & 4.41 \\
\hline 11 & - & - & - & 5.96 \\
\hline Mean & 4.10 & 4.27 & 6.36 & 4.37 \\
\hline S. D. & 1.06 & 0.92 & 3.07 & 0.93 \\
\hline
\end{tabular}

As shown in Figs.2. and 3., the methodology can be applied not only to random numbers but also to actual data collected from construction sites. Table 5. shows $\mu$ " and $\sigma$ " for each step, and compares the value with the mean and standard deviation of each dataset. That is, the productivity data and the actual data were analyzed using the same methodology, and the results are shown in Tables 3. and 5., respectively.

Using the mean value could lead to serious mistakes in process management. For this specific construction site, the mean of the third dataset has a high productivity value of 6.36 units/h. However, using the Bayesian methodology, the productivity converges to 4.44 units/h after considering datasets 1 through 3 . The standard deviation also varies and has a unique value for each dataset. However, when using the Bayesian methodology, the value decreases as more data are added. The application of Bayesian posterior probability in analyzing construction productivity gives

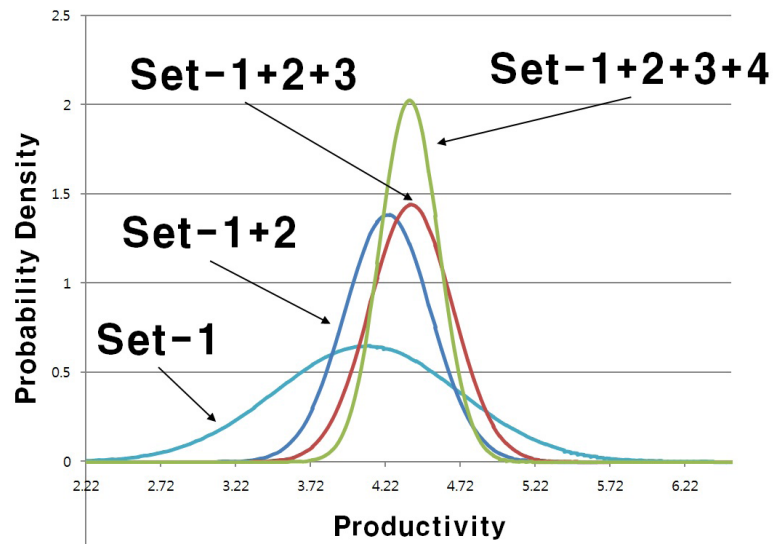

Fig.3. Normal Distribution of Actual Data

Table 5. $\mu$ " and $\sigma$ " Calculation and Comparison Using Actual Data (units/h)

\begin{tabular}{c|c|c|c|c}
\hline Description & Set 1 & Set 2 & Set 3 & Set 4 \\
\hline$\mu^{\prime \prime}$ & 4.10 & 4.26 & 4.44 & 4.40 \\
\hline Mean & 4.10 & 4.27 & 6.36 & 4.37 \\
\hline$\sigma^{\prime \prime}$ & 1.06 & 0.31 & 0.30 & 0.20 \\
\hline S. D. & 1.06 & 0.92 & 3.07 & 0.93 \\
\hline
\end{tabular}

a reliable result. It is expected that this methodology can be used to improve the productivity management for a construction site by applying the methodology to various operations with a larger number of datasets. Table 6. shows the overall analysis results of the actual data between the case in which mean values and standard deviations are calculated as historical datasets and that in which the Bayesian posterior approach is applied.

Table 6. Overall Analysis Results between Simple Average Calculation and Bayesian Application (units/h)

\begin{tabular}{c|c|c|c|c}
\hline Description & Set 1 & Set $1+2$ & Set $1+2+3$ & Set $1+2+3+4$ \\
\hline$\mu^{\prime}$ & 4.10 & 4.26 & 4.44 & 4.40 \\
\hline Mean & 4.10 & 4.22 & 5.18 & 4.89 \\
\hline$\sigma^{\prime}$ & 1.06 & 0.31 & 0.30 & 0.20 \\
\hline S. D. & 1.06 & 0.91 & 2.36 & 1.99 \\
\hline
\end{tabular}

In this chapter, the analysis process has been performed using Microsoft Excel 2010. It has been analyzed that systemization of the analysis process is mandatory considering application in the construction site. The overall process of specific spreadsheet implementation considering practical application of the Bayesian method is shown in Fig.4.

\section{Database Implementation}

The research by Han et al. (2014) suggested the basic framework of the database with various functions that site personnel can acquire information from with ease. The development of the database framework focuses on a way to apply the Bayesian approach to a database, while designing for users' convenience.

Construction data storage systems used in an active construction site were studied by interviewing the engineers and the site manager. The productivity 


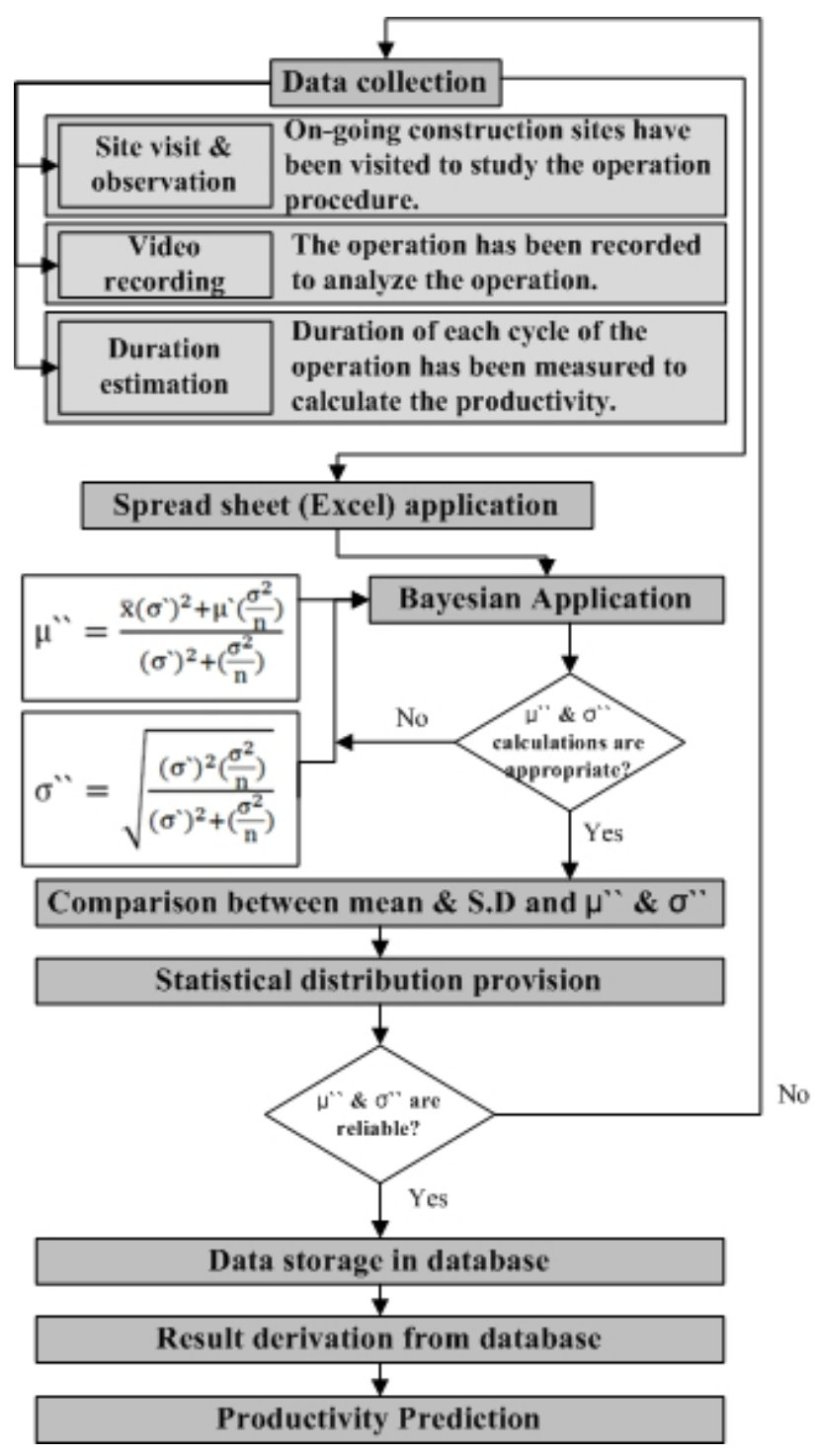

Fig.4. Practical Procedure for Bayesian Application

data were stored in a Microsoft Excel file in which the number of laborers that participated in a specific operation was recorded every day. Additionally, the quantity of the work done each day was also recorded. The site engineers calculated and recorded the daily productivity. However, there are no systems currently available to forecast or analyze productivity. Therefore, this paper suggests a methodology for developing a database using the Bayesian posterior probability described in Chapters 2.2 and 2.4. The user defines the data to be recorded (e.g., laborer working hours per day, work done per day, duration of each unit process, etc.). The user also defines a formula for the result (e.g., daily productivity, hourly productivity, unit cost, etc.). For example, the hourly productivity is defined as in equation (6).

$$
\text { Hourly Prod. }\left[\frac{\text { unit }}{\text { hour }}\right]=\frac{\text { work per day [units }]}{\text { working hours per day }[8 \mathrm{~h}]}
$$

The user can decide the number of datum to be used in one dataset. If the user decides to define a weekly dataset, there will be five or seven datum in the dataset, depending on the number of working days in one week. The application of Bayesian posterior probability to the database enables the inclusion of past data and manages the productivity. The database shows the user how the productivity changes and the value to which it appears to be converging. The database suggested in this paper, as shown in Figs.5. and 6., enables integrated construction data management. Fig.5. shows the input screen of the database. The data are inserted into the database as shown in the highlighted part in Fig.5. The output screen of the database is shown in Fig.6. The database automatically shows the histogram of the data such that the user will be able to view the overall distribution of the data before analysis. Application of the Bayesian posterior probability approach in the database automatically analyzes the input data and suggests an optimal value of productivity. The highlighted area in Fig.6. shows the optimal value of productivity deduced by the application of Bayesian methodology. In the curtain wall operation, for example, if the engineers decide to record the number of installed units daily, record 6 units/day, and there are 8 working hours in a day, the hourly productivity data will be computed as follows.

$$
\frac{6 \text { units }}{\text { day }} * \frac{\text { day }}{8 \mathrm{~h}}=\frac{0.75 \text { units }}{\text { hour }}
$$

The productivity was calculated based on the installation of each unit. If the installation duration of one unit is $20 \mathrm{~min}$, the hourly productivity will be

$$
\frac{1 \mathrm{unit}}{20 \mathrm{~min}} * \frac{60 \mathrm{~min}}{1 \mathrm{~h}}=3 \frac{\text { units }}{\mathrm{h}}
$$

Recording more datasets will automatically generate a distribution of the data by applying the Bayesian posterior probability. The proposed method is expected to improve productivity management, and can be used to monitor numerous repetitive operations in high-rise construction projects.

\section{Conclusion}

The curtain wall operation represents $10 \%$ to $15 \%$ of the total construction cost of a construction project, and the operation is generally part of the critical path. Therefore, the paper suggests a productivity analysis and forecasting methodology focusing on curtain wall operations.

In the academia of construction or civil engineering, a few studies where Bayesian statistical methodology was adopted have been released so far. These studies were mostly focused on illustrating updated situations especially on experimental data. However, this study suggested the application of this method for predicting the feasible construction performance of which attributes were totally different with experimental information. Also, the suggested database prototype in this study would be beneficial for developing advanced 


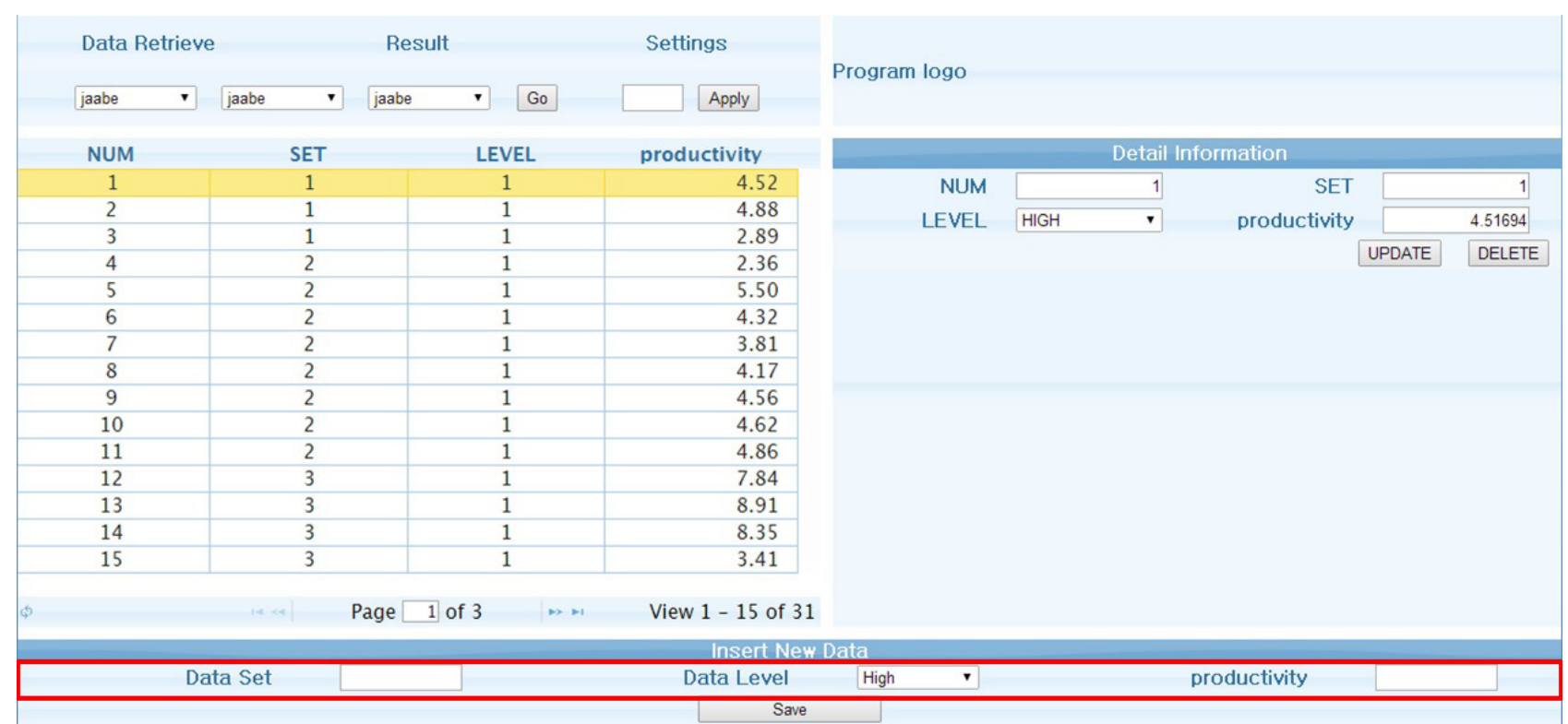

Fig.5. Input Screen in the Database

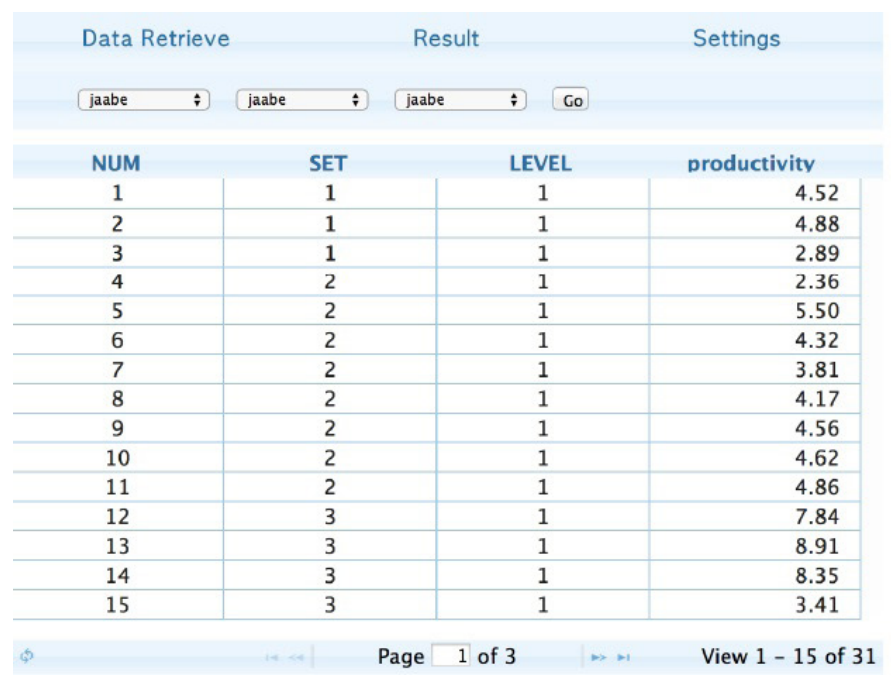

Program logo

Fig.6. Output Screen in the Database

feasible systems where similar statistical methods are implemented in the future. The Bayesian posterior probability was implemented using random data at first in this paper. The reason for this was to investigate the feasibility of the methodology to satisfy the research objectives and to explore the possibility of expanding it to a wider range of applications. The proposed methodology was implemented with actual data collected from an ongoing construction project, after establishing the validity of the pre-test. The results show that $\mu$ " converges to an optimal value when using the Bayesian methodology. However, the traditional use of the mean value indicates that using one dataset could lead to incorrect decision making. Moreover, it has been determined that as more datasets were recorded, the value of $\sigma$ " was lower than the standard deviation of each dataset. The proposed methodology enables us to forecast the optimal productivity value of the current operation that is being analyzed. The methodology can be applied in productivity analysis, and in cost calculations. The unit cost data can be calculated and recorded in the database, where the optimal value of the unit cost is automatically calculated and the distributions are displayed. The contributions of this paper can be identified as two: academic and industrial. This paper suggested the application of Bayesian methodology to productivity analysis in construction where a large amount of raw data has been generated with various attributes. The detailed application procedure with significant results would assist researchers in academia to investigate and implement relevant research methodologies. Regarding contributions to industry, the suggested prototype of a database for productivity analysis enables practitioners to develop similar knowledge systems which are capable of delivering valuable information and making proper decisions prior to actual works in construction operations. 
Moreover, this methodology could be used in other repetitive operations, especially in high-rise construction projects. The additional supplement of the Bayesian probabilistic approach to the database enables users to acquire results despite frequent updates to the data. It is expected that this system will be of significant use to site personnel. Four datasets have been used; however, the actual quantity of data used in this study was less than that generally used in statistical studies. In future studies, there is a need to collect more data to achieve more reliable results.

\section{Acknowledgement}

This research was supported by the Basic Science Research Program through the National Research Foundation of Korea (NRF), funded by the Ministry of Education, Science and Technology (MEST) of the Korean government (no. NRF2012R1A2A2A01046193). The authors gratefully acknowledge this support.

\section{References}

1) Ang, A and Tang, W. (2007) Probability concepts in engineering. 2nd ed. Hoboken: John Wiley \& Sons, Inc.

2) Al-Sabah, S., Jaber, A., Fereig, S. and Hoare, D. (2003) A database management system to document and analyze construction claims. Advances in Engineering Software. 34(8), pp.477-491.

3) Benjamin, J. (1970) Probability statistics, and decision for civil engineers. New York: McGraw-Hill, Incorporated.

4) Choi, S. (2006) A Study on Scheme of Execution in Curtain Wall work for the Application of Supply Chain Management [Master dissertation]. Seoul: Soongsil University.

5) Christian, J. (2004) Geotechnical Engineering Reliability: How Well Do We Know What We Are Doing? Journal of Geotechnical and Geoenvironmental Engineering. 130(10), pp.985-1003.

6) Forcada, N., Casals, M., Roca, X. and Gangolells, M. (2007) Adoption of web databases for document management in SMEs of the construction sector in Spain. Automation in Construction. 16(4), pp.411-424.

7) Gardoni, P., Nemati, K. and Noguchi, T. (2007) Bayesian Statistical Framework to Construct Probabilistic Models for the Elastic Modulus of Concrete. Journal of Materials in Civil Engineering ASCE. 19(10), pp.898-905.

8) Han, S. (2010) Productivity Analysis Comparison of Different Types of Earthmoving Operations by Means of Various Productivity Measurements. Journal of Asian Architecture and Building Engineering, 9(1), pp.185-192.

9) Huang, J. and McBean, E. (2008) Using Bayesian statistics to estimate chlorine wall decay coefficients for water supply system. Journal of Water Resources Planning and Management ASCE. 134(2), pp.129-137.

10) Han, S., Lee, T. and Ko, Y. (2014) Implementation of Construction Performance Database Prototype for Curtain Wall Operation in High-Rise Building Construction, Journal of Asian Architecture and Building Engineering, 13(1), pp.149-156.

11) Johnson, R. and Wichern, D. (2007) Applied multivariate statistical analysis. 6th ed. Upper Saddle River: Pearson Education, Inc.

12) Kang, J. (2011) Prediction of Shear Strength of Reinforced Concrete Beams by Probabilistic Approach [Master dissertation]. Seoul: University of Seoul.

13) Kim, H. (2012) Fuzzy Technique-based Productivity Estimation by means of Construction Delay Factor Analysis on Curtain wall Operations in High-rise Building Constructions [Master dissertation] Incheon: Inha University.
14) Kim, B. and Reinschmidt, K. (2009) Probabilistic forecasting of project duration using Bayesian inference and the beta distribution. Journal of Construction Engineering and Management. 135(3), pp.178-186.

15) Kim, D. Kim, B. (2010) Construction Robot System Design for High-rise Building External wall Maintenance. Proceedings of the Korea Institute of Building Construction (Industry). 10 (2), pp.710 .

16) Lim, J. (2009) Development of Duration Estimation Forecasting Model for Curtain Wall Construction: focused on Unit System Method [Master dissertation]. Seoul: University of Seoul.

17) Leu, S. and Chang, C. (2013) Bayesian-network-based safety risk assessment for steel construction projects. Accident Analysis \& Prevention. 54, pp.122-133.

18) Lee, E., Park, Y. and Shin, J. (2009) Large engineering project risk management using a Bayesian belief network. Expert Systems with Applications. 36 (3), pp.5880-5887.

19) Labuz, E., Chang, M. and Pakzad, S. (2010) Local Damage Detection in Beam-Column Connections Using a Dense Sensor Network. Proc. of 19th Annual Structures Congress ASCE. pp.3143-3154

20) Lee, T., Kim, H., Ko, Y. and Han, S. (2011) Quantitative comparisons of construction productivity of curtain wall operations in high-rise buildings by means of simulation techniques. Advanced Materials research. 250, pp.3577-3580.

21) Navon, R. (2005) Automated project performance control of construction projects. Automation in Construction. 14, pp.467476.

22) Son, Y. (2002) The Study of Construction Method of Curtain Wall System by the Case Study of Construction Site Application [Master dissertation]. Seoul: Hanyang University.

23) Soibelman, L. and Kim, H. (2002) Data Preparation Process for Construction Knowledge Generation through Knowledge Discovery in Databases. J. Comput. Civ. Eng. 16(1), pp.39-48.

24) The Korean Ministry of Land Transport and maritime Affairs. (2011) Construction Statistics. South Korea.

25) Truong, L. (2009) Quantifying schedule risk in construction projects using Bayesian belief networks. International Journal of Project Management. 27(1), pp.39-50.

26) Tah, J., Carr, M. and Howes, R. (1999) Information modeling for case-based construction planning of highway bridge projects. Advances in Engineering Software. 30(7), pp.495-509.

27) Yuen, K. (2010) Bayesian Methods for Structural Dynamics and Civil Engineering. Singapore: John Wiley \& Son. 